\title{
Numerical investigation on natural convection in asymmetric channel-chimney systems
}

\author{
A. Andreozzi ${ }^{1}$, B. Buonomo ${ }^{2}$ \& O. Manca $^{2}$ \\ ${ }^{1}$ DETEC, Università di Napoli FEDERICO II, Napoli, Italy \\ ${ }^{2}$ DIAM, Seconda Università di Napoli, Aversa (CE), Italy
}

\begin{abstract}
A numerical investigation on two-dimensional transient natural convection in a vertical channel-chimney system with the channel heated asymmetrically and the chimney geometrically asymmetric is carried out. The heated channel wall is at uniform heat flux and the other one is assumed to be adiabatic. The chimney walls are adiabatic. Results are obtained for different expansion and extension ratios of the channel-chimney system and Rayleigh number equal to $10^{4}$ and $10^{6}$. They are presented in terms of maximum heated wall temperatures and average Nusselt number as a function of time and stream function fields for some time values. Optimal thermal configurations in terms of minimum value of maximum heated wall temperature and maximum average Nusselt numbers are evaluated. Complex flow structures in the channel-chimney system are detected.

Keywords: natural convection, numerical investigation, asymmetric channelchimney systems.
\end{abstract}

\section{Introduction}

More recent trends in natural convection research are to find new configurations to improve the heat transfer parameters or to analyze standard configurations to carry out optimal geometrical parameters for a higher heat transfer rate and the transient behavior for a suitable thermal design. A very simple method, which allows to improve the chimney effect and consequently heat transfer rate in vertical channels and other configurations, is to place parallel adiabatic extensions downstream heated configurations $[1,2]$.

A first research on the chimney effects was obtained by Haaland and Sparrow [2]. A vertical channel with point source or distributed heat source situated at 
channel inlet was investigated. A numerical study on the natural convection in an isothermal vertical parallel-plates with straight adiabatic downstream extensions was carried out in [3]. A numerical investigation on unheated chimney attached to a vertical isothermal tube was accomplished in [4]. A numerical study on isoflux channels using the elliptic form of the governing equations was conducted in [5]. A composite I-shaped computational domain was employed in order to obtain a more realistic model. A numerical simulation of a channel-chimney system was carried out in [6]. How and why the "chimney effect" worsens was emphasized. It was connected to the cold inflow at the outlet section and this effect was more stressed at higher Rayleigh number. Numerical and experimental flow visualizations were carried out in [7]. Results were presented in terms of stream function and temperature fields for different expansion and extension ratios. An experimental investigation on air natural convection in a vertical channel asymmetrically heated at uniform heat flux, with downstream unheated parallel extensions, was carried out in [8]. One extension was coplanar to the unheated channel wall and the distance between the extensions was equal to or greater than the channel gap. Optimal configurations of the system with asymmetrical chimney were detected.

It seems that numerical investigations on transient natural convection in vertical channels have been carried out only for simple channel configurations [9-12] and vertical symmetrical channel-chimney system with the channel heated symmetrically [13]. Then, there is a lack of investigations on transient natural convection in vertical asymmetrical channel-chimney system heated asymmetrically.

In this paper a numerical simulation for two-dimensional, laminar and transient regime is carried out in an asymmetric channel-chimney system. The computational domain is made up of the physical configuration and two reservoirs placed upstream and downstream the channel-chimney system. The numerical analysis is obtained by means of the commercial Fluent code. Results are obtained for different expansion and extension ratios of the channel-chimney system and Rayleigh number. They are presented in terms of stream function fields for some times, maximum heated wall temperatures and average Nusselt number as a function of time.

\section{Mathematical description and numerical procedure}

The aim of this paper is the transient numerical analysis of natural convection in air in an asymmetric channel-chimney system. The investigated geometry is depicted in Fig. 1(a). It is made up of a vertical channel with two parallel plates, one plate is heated with uniform heat flux $\dot{q}$ and the other one is adiabatic; the height of the heated plate is $L_{h}$ whereas the channel gap is $b$. On the top of the channel, there is a chimney made up of two insulated parallel and vertical plates; their height is $L-L_{h}$ and the distance between them is $B$. An enlarged computational domain has been chosen. It is made up of the previously described geometry and of two reservoirs of height $L_{x}$ and width $L_{y}$, which are placed upstream of the channel and downstream 


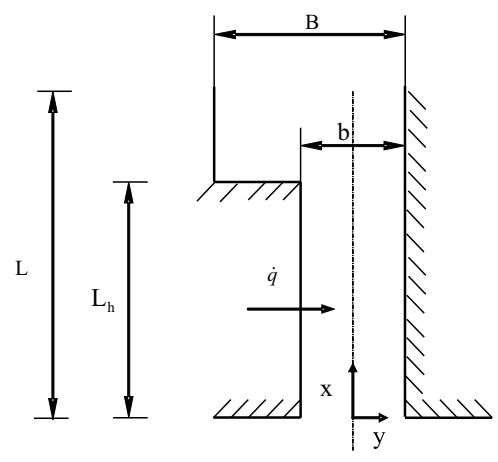

(a)

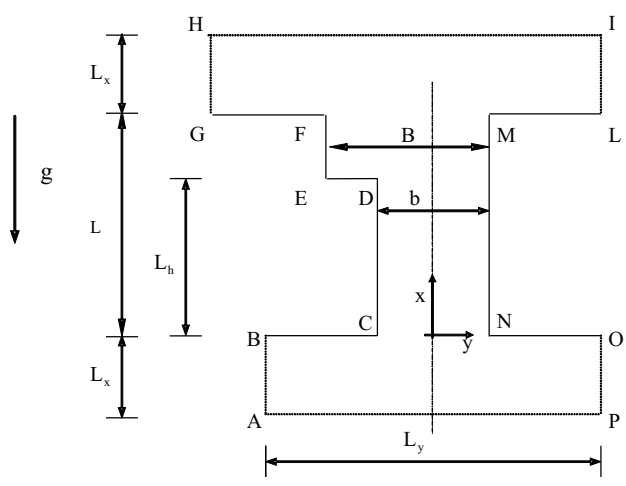

(b)

Figure 1: Sketch of the system: (a) physical domain; (b) computational domain.

of the chimney. The employed computational domain is shown in Fig. 1(b). All thermophysical properties of the fluid are assumed to be constant with temperature except for the density, as suggested by the Boussinesq approximation. With the above assumptions, the governing equations are in dimensionless form:

$$
\begin{gathered}
\frac{\partial U}{\partial X}+\frac{\partial V}{\partial Y}=0 \\
\frac{\partial U}{\partial \tau}+\frac{\partial U^{2}}{\partial X}+\frac{\partial(U V)}{\partial Y}=-\frac{\partial P}{\partial X}+\left[\frac{\partial^{2} U}{\partial X^{2}}+\frac{\partial^{2} U}{\partial Y^{2}}\right] \\
\frac{\partial V}{\partial \tau}+\frac{\partial(U V)}{\partial X}+\frac{\partial V^{2}}{\partial Y}=-\frac{\partial P}{\partial Y}+\left[\frac{\partial^{2} V}{\partial X^{2}}+\frac{\partial^{2} V}{\partial Y^{2}}\right]+G r \theta \\
\frac{\partial \theta}{\partial \tau}+\frac{\partial(U \theta)}{\partial X}+\frac{\partial(V \theta)}{\partial Y}=\frac{1}{\operatorname{Pr}}\left[\frac{\partial^{2} \theta}{\partial X^{2}}+\frac{\partial^{2} \theta}{\partial Y^{2}}\right]
\end{gathered}
$$

where the dimensionless variables are defined as:

$$
\begin{gathered}
X=x / b, \quad Y=y / b, \quad \tau=(t \nu) / b^{2}, \\
U=u b / \nu, \quad V=v b / \nu, \\
P=\left(p-p_{\infty}\right) b^{2} /\left(\rho \nu^{2}\right), \quad \theta=\left(T-T_{\infty}\right) k /(\dot{q} b), \\
G r=g \beta \dot{q} b^{4} /\left(k \nu^{2}\right), \quad \operatorname{Pr}=\nu / a, \quad R a=G r \operatorname{Pr}
\end{gathered}
$$

In Eq.(4) the terms of viscous dissipation and of pressure work are neglected. 
Table 1: Boundary conditions.

\begin{tabular}{|c|c|}
\hline Zone & Boundary conditions \\
\hline \hline AB, OP, PA & Pressure Inlet \\
\hline GH, HI, IL & Pressure Outlet \\
\hline CD & No slip condition, $\frac{\partial \theta}{\partial Y}=-1$ \\
\hline BC, DE, FG, LM, NO & No slip condition, $\frac{\partial \theta}{\partial X}=0$ \\
\hline EF, MN & No slip condition, $\frac{\partial \theta}{\partial Y}=0$ \\
\hline
\end{tabular}

The average Nusselt number is defined as:

$$
N u=\frac{b}{L_{h}} \int_{0}^{L_{h} / b} \frac{d X}{\theta_{w}(X)}
$$

With reference to Fig. 1(b), the imposed boundary conditions are reported in Table 1.

The commercial Fluent CFD code was employed to solve the governing equations. The SIMPLE scheme was chosen to couple pressure and velocity. The converging criteria were $10^{-6}$ for the residuals of the velocity components and $10^{-8}$ for the residuals of the energy. The time at which steady state or quasi steady state conditions are assumed is denoted as $\tau_{s s}$.

A preliminary study has been performed to determine an adequate grid to ensure grid independence of the main variables such as the average Nusselt number, $N u$. Computations have been made for the flow corresponding to $R a=10^{4}$, a time step equal to $\Delta \tau=10^{-3}$, for a channel aspect ratio $L_{h} / b$ equal to $10, L / L_{h}=1.5$ and $B / b=4.0$ on a grid consisting of $35 \times 11,71 \times 21,141 \times 41$ and $281 \times 81$ grid points inside the vertical channel. The percentage discrepancy between the average Nusselt number value on a grid consisting of $71 \times 21$ and an asymptotic value obtained by means of the Richardson extrapolation is less than $1.5 \%$. So, the results reported in this paper have been obtained with a $71 \times 21$ grid which ensured a good compromise between the computational time and accuracy.

A comparison among the $N u$ values for four different time steps, $\Delta \tau=10^{-2}$, $10^{-3}, 10^{-4}$ and $10^{-5}$, has been carried out for the $71 \times 21$ mesh for the configuration with $L_{h} / b=10, L / L_{h}=1.5$ and $B / b=4.0$ at different Rayleigh number values. The time step in the transient calculations is fixed at $\Delta \tau=10^{-3}$ for $R a$ in the range $\left[10^{2}-10^{4}\right]$, with a maximum percentage discrepancy equal to $0.1 \%$, and $\Delta \tau=10^{-4}$ for $R a=10^{5}$ and $10^{6}$, with a maximum percentage discrepancy equal to $0.5 \%$. 


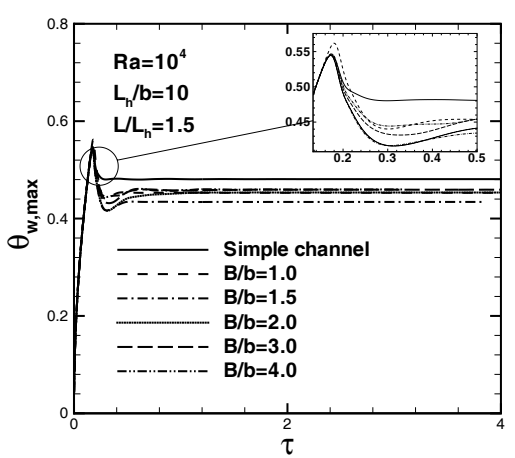

(a)

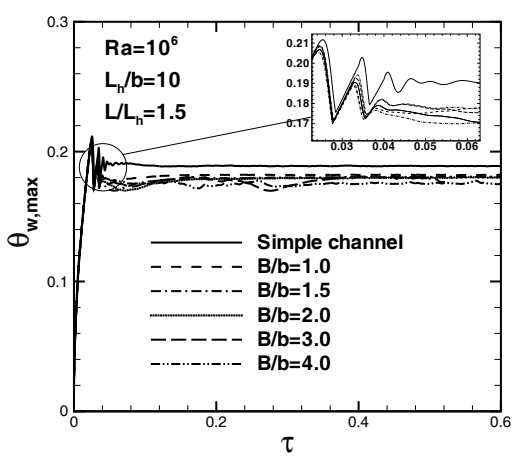

(b)

Figure 2: Maximum heated wall temperature as a function of time at different expansion ratio values, $L_{h} / b=10$ and $L / L_{h}=1.5$ for: (a) $R a=10^{4}$ and (b) $R a=10^{6}$.

An analogous analysis has been carried out to set the optimal reservoirs dimensions, $L_{x}$ and $L_{y}$. A reservoir horizontal dimension, $L_{y}$, equal to eleven times $b$ and a reservoir vertical dimension, $L_{x}$, equal to the heated plate length $L_{h}$ have been chosen.

\section{Results and discussion}

In the following results are presented for air, for a channel aspect ratio $L_{h} / b=10$, for an extension ratio $L / L_{h}=1.5$ and 2.0 and for an expansion ratio $B / b=1.0$, 1.5, 2.0, 3.0 and 4.0. In the following comparison with data related to simple channel are given. All data are in dimensionless form and the results are showed for $R a=10^{4}$ and $10^{6}$.

Maximum heated wall temperature as a function of the time for $R a=10^{4}$ and $10^{6}$ and $B / b=1.0,1.5,2.0,3.0$ and 4.0 and the simple channel is reported in Figs. 2 and 3 for $L / L_{h}=1.5$ and 2.0, respectively. In all cases, maximum wall temperature increases as the time increases up to a maximum value, which is attained at smaller time value as larger the $R a$ value is. When the maximum wall temperature profile has attained the maximum with respect to the time, its value decreases and the maximum temporal value is an overshoot. The overshoot in wall temperature profile is typical of the natural convection problem in configuration with walls heated at assigned heat flux. For simple channel the maximum wall temperature at steady state is the greatest value among the different cases for different $B / b$ values, resulting the most critical configuration at the steady state condition, according with [6]. However, it is interesting to observe that for 
$L / L_{h}=1.5$ and $R a=10^{4}$, in Fig. 2(a), the maximum value for $B / b=1.0$ is the greatest whereas the simple channel presents the overshoot almost equal to the other configurations. This result for asymmetric geometrical and heated channelchimney system is in agreement with the results for symmetric geometrical and heated channel-chimney system [13]. For $R a=10^{4}$, in Figs. 2(a) and 3(a), after the overshoot the temperature value decreases until it attains a minimum value, except for the simple channel. Moreover, the best configuration at the steady state, in terms of minimum value of the maximum wall temperature, is obtained for $B / b=1.5$. For $L / L_{h}=2.0$ the configuration with $B / b=4.0$ presents some oscillations due to the downflow or cold inflow of the fluid in the channel-chimney system which allows the penetration of cold air from the outlet section of the system in the chimney. The cold inflow determines a periodic flow for this configuration. This is in agreement with [14]. For $R a=10^{6}$ heated wall temperature profiles, in Figs. 2(b) and 3(b), after the first overshoot and undershoot, show damped oscillations for the simple channel and $B / b \leq 3.0$. For $B / b=4.0$ the oscillations are almost constant in the time but the average profiles is constant, i. e., the profile oscillates around a quasi steady state value of the maximum wall temperature. For this Ra value equal to $10^{6}$, the best configuration at steady or quasi-steady state is obtained for $B / b=4.0$. This means that at steady or quasi-steady state for $R a=10^{6}$, in the range of the considered expansion ratio, a greater cold inflow in an asymmetric heated channel-chimney system allows an enhancement of the heat transfer in the channel whereas for the symmetric heated channel-chimney system the cold inflow determines a decrease of the mass flow rate and consequently of the heat transfer in the channel [14]. In Figs. 2 and 3 it is observed that increasing the extension ratio, $L / L_{h}$, the maximum heated wall temperature values at steady or quasi-steady state decrease according with the increase of the mass flow rate due to the greater buoyancy force.

In Fig. 4 the Nusselt number as a function of time is reported for $R a=10^{6}$ and $L / L_{h}=1.5$ and 2.0. The trends of the profiles confirm the oscillations for this Rayleigh number value. The configurations for $B / b=4.0$ present the highest Nusselt number values among the considered configurations for both the extension ratios. They correspond to the best configurations also in terms of the heat transfer coefficients. Moreover, the percent increase of the Nusselt number between $L / L_{h}=1.5$ and $L / L_{h}=2.0$ for $B / b=4.0$ at quasi-steady state is about $25 \%$.

Stream function fields for $R a=10^{6}, L / L_{h}=2.0$ and $B / b=2.0$ and 4.0 at some times are given in Figs. 5 and 6 . For the smaller considered $B / b$ at $\tau=\tau_{s s} / 50$, in Fig. 5(a), the fluid flows inside the channel adjacent to the heated wall whereas close to the opposite adiabatic wall, the shroud, a sort of vortex cell is present. This cell is extended in the chimney up to half height of the chimney. For $\tau \geq \tau_{s s} / 5$, in Figs. 5(b)-5(d), the fluid enters in the channel and the vortex is moved completely in the chimney. For $\tau=\tau_{s s} / 5$, in Fig. 5(b), a cold inflow coming from the outlet section of the chimney goes in the chimney and a vortex cell is present close to the adiabatic wall in the upper part of the chimney. The vortex cell for $\tau=\tau_{s s} / 2$, in Fig. 5(c), expands inside the chimney and there is 


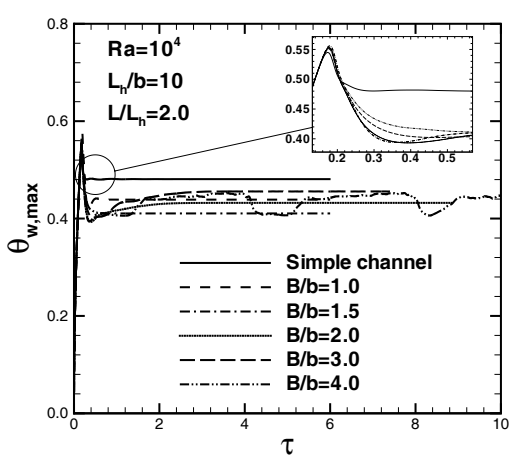

(a)

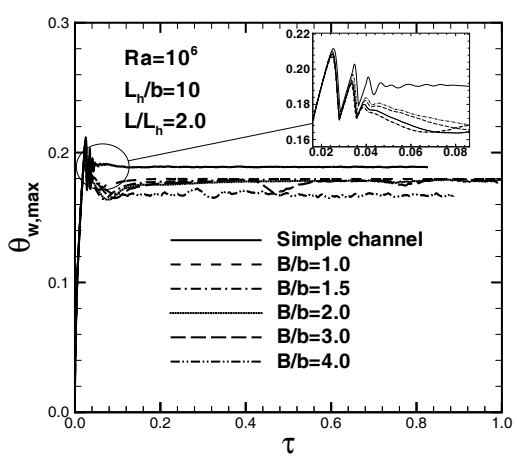

(b)

Figure 3: Maximum heated wall temperature as a function of time at different expansion ratio values, $L_{h} / b=10$ and $L / L_{h}=2.0$ for: (a) $R a=10^{4}$ and (b) $R a=10^{6}$.

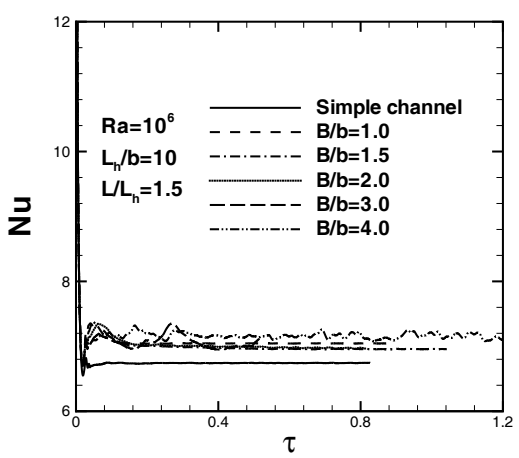

(a)

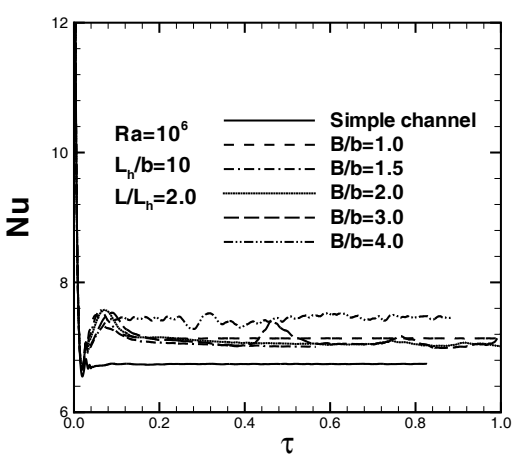

(b)

Figure 4: Average Nusselt number as a function of time at different expansion ratio values, $R a=10^{6}$ and $L_{h} / b=10$ for: (a) $L / L_{h}=1.5$ and (b) $L / L_{h}=2.0$.

not cold inflow. For $\tau=\tau_{s s}=0.8$, in Fig. 5(d), the cold inflow is extended in the greater part of the chimney and it penetrates in the channel. The vortex cell is squashed on the higher part of the shroud. For $B / b=4.0$ at $\tau=\tau_{s s} / 50$, in Fig. 6(a), two cells are present, one in the channel and one in the lower part of the chimney close to the channel outlet section on the heated wall side. Increasing the time, Figs. 6(b)-6(d), the cell in the channel disappears whereas vortices are present in the lower corner of the chimney and adjacent to the adiabatic shroud. 


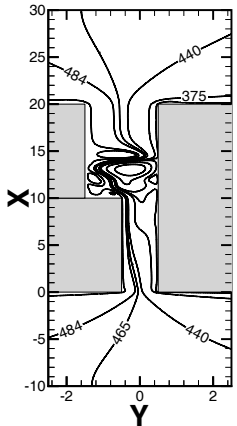

(a)

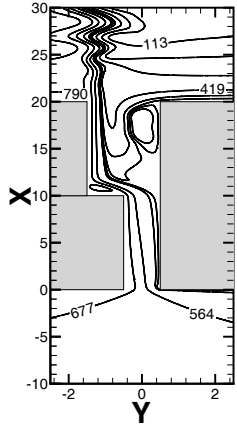

(b)

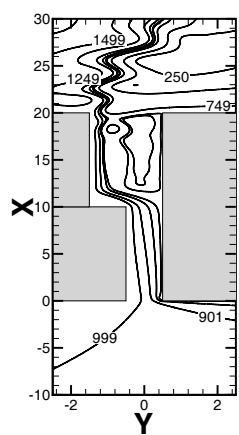

(c)

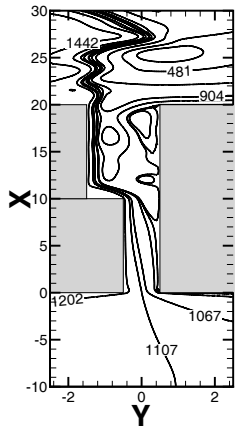

(d)

Figure 5: Stream function fields for $R a=10^{6}, L / L_{h}=2.0$ and $B / b=2.0$ at: (a) $\tau=\tau_{s s} / 50$, (b) $\tau=\tau_{s s} / 5$, (c) $\tau=\tau_{s s} / 2$ and (d) $\tau=\tau_{s s}$.

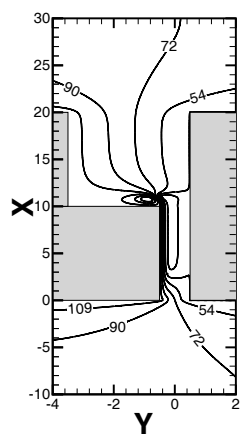

(a)

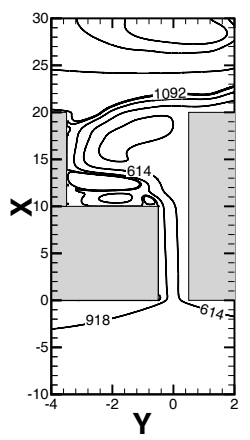

(b)

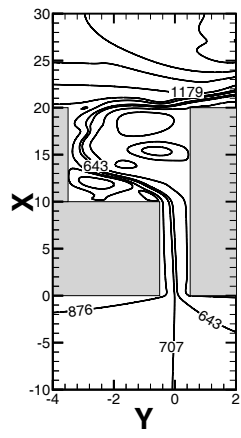

(c)

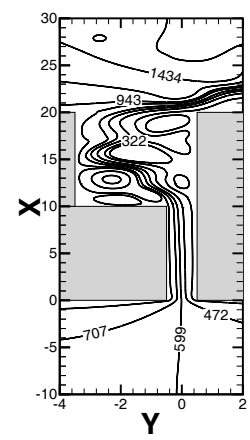

(d)

Figure 6: Stream function fields for $R a=10^{6}, L / L_{h}=2.0$ and $B / b=4.0$ at: (a) $\tau=\tau_{s s} / 50$, (b) $\tau=\tau_{s s} / 5$, (c) $\tau=\tau_{s s} / 2$ and (d) $\tau=\tau_{s s}$.

\section{Conclusions}

Transient natural convection in air in a vertical asymmetrical channel-chimney system asymmetrically heated was numerically investigated. The channel heated wall was considered at uniform heat flux and results allowed to detect the different fluid dynamic and thermal behaviors for different expansion and extension ratios. The effect of two Rayleigh number $\left(10^{4}\right.$ and $\left.10^{6}\right)$ was accomplished in order to detect the complex flow structures in the channel-chimney system. For highest $\mathrm{Ra}$ and expansion ratio, heated wall temperature profiles as a function of time oscillated. The quasi-steady state of the maximum wall temperature was the lowest 
for the highest expansion ratio $(B / b=4.0)$ whereas for $R a=10^{4}$ the minimum value of the maximum heated wall temperature was attained for $B / b=2.0$. The same results were obtained also for the average Nusselt numbers. Stream function fields allowed to visualize the flow structures inside the channel and the chimney. A cold inflow was detected together with some vortex cells inside the chimney.

\section{Nomenclature}

$a$
$b$
$B$
$g$
$G r$
$k$
$L$
$L_{h}$
$L_{x}$
$L_{y}$
$N u$
$p$
$P$
$P r$
$\dot{q}$
$R a$
$t$
$T$
$u, v$
$U, V$
$x, y$
$X, Y$
Greek symbols
$\beta$
$\nu$
$\theta$
$\rho$
$\tau$

thermal diffusivity, $\mathrm{m}^{2} / \mathrm{s}$

channel gap, $m$

chimney gap, $m$

acceleration due to gravity, $\mathrm{m} / \mathrm{s}^{2}$

Grashof number, Eq.(5)

thermal conductivity, $W / m K$

system height, $m$

channel height, $m$

height of reservoir, $m$

width of reservoir, $m$

average Nusselt number, Eq.(6)

pressure, $P a$

dimensionless pressure, Eq.(5)

Prandtl number, Eq.(5)

heat flux, $W / m^{2}$

Rayleigh number, Eq.(5)

time, $s$

temperature, $K$

velocity components, $\mathrm{m} / \mathrm{s}$

dimensionless velocity components, Eq.(5)

Cartesian coordinates, $m$

dimensionless Cartesian coordinates, Eq.(5)

Subscripts

$\infty$

volumetric coefficient of expansion, $1 / K$

kinematic viscosity, $\mathrm{m}^{2} / \mathrm{s}$

dimensionless temperature, Eq.(5)

density, $\mathrm{kg} / \mathrm{m}^{3}$

dimensionless time, Eq.(5)

$\max$

free stream condition

$s s$

maximum value

steady state

wall

\section{Acknowledgements}

This work was supported by MIUR with a 2005 PRIN grant. 


\section{References}

[1] Manca, O., Morrone, B., Nardini, S. \& Naso, V., Computational Analysis of Convection Heat Transfer, WIT Press: Southampton, Boston, chapter Natural Convection in Open Channels, 2000.

[2] Haaland, S.E. \& Sparrow, E.M., Solutions for the Channel Plume and the Parallel-Walled Chimney. Numerical Heat Transfer, 6, pp. 155-172, 1983.

[3] Oosthuizen, P.H., A Numerical Study of Laminar Free Convective Flow through a Vertical Open Partially Heated Plane Duct. ASME HTD Fundamentals of Natural Convection - Electronic Equipment Cooling, 32, pp. 41-48, 1984.

[4] Asako, Y., Nakamura, H. \& Faghri, M., Natural Convection in a Vertical Heated Tube Attached to a Thermally Insulated Chimney of a Different Diameter. ASME Journal of Heat Transfer, 112, pp. 790-795, 1990.

[5] Campo, A., Manca, O. \& Morrone, B., Numerical Analysis of Partially Heated Vertical Parallel Plates in Natural Convective Cooling. Numerical Heat Transfer, Part A, 36, pp. 129-151, 1999.

[6] Andreozzi, A., Buonomo, B. \& Manca, O., Numerical study of natural convection in vertical channels with adiabatic extensions downstream. Numerical Heat Transfer, Part A, 47, pp. 1-22, 2005.

[7] Andreozzi, A., Buonomo, B., Manca, O. \& Musto, M., Flow Visualization of Natural Convection in Asymmetric Channel - Chimney Systems. Proc. of Seventh Triennal International Symposium on Fluid Control, Measurement and Visualization - FLUCOME, paper ID-127, Sorrento, Italy, 2003.

[8] Manca, O., Musto, M. \& Naso, V., Experimental investigation of natural convection in an asymmetrically heated vertical channel with an asymmetric chimney. ASME Journal of Heat Transfer, 127, pp. 888-896, 2005.

[9] Joshi, H.M., Transient Effects in Natural Convection Cooling of Vertical Parallel Plates. Int Communications in Heat and Mass Transfer, 15 (2), pp. 227-238, 1988.

[10] Chang, B. \& Lin, K., Transient Buoyancy-Induced Flow through a Heated, Vertical Channel of Finite Height. Numerical Heat Transfer, Part A, 16, pp. 15-35, 1989.

[11] Chang, K.P. \& Hung, Y.H., Transient natural convection between vertical finite length heated plates. Journal of Thermophysics and Heat Transfer, 11 (2), pp. 203-211, 1997.

[12] Andreozzi, A., Buonomo, B. \& Manca, O., Numerical study of transient natural convection in vertical asymmetrically heated channels. Atti del $61^{\circ}$ Congresso ATI, Perugia, Italy, 1, pp. 459-464, 2006.

[13] Andreozzi, A., Buonomo, B. \& Manca, O., Numerical simulation of transient natural convection in a channel-chimney system. Proc. HT2005, paper HT2005-72628, San Francisco, California, USA, 2005.

[14] Fisher, T.S. \& Torrance, K.E., Experiments on Chimney-Enhanced Free Convection. ASME Journal of Heat Transfer, 121, pp. 607-608, 1999. 